\title{
HIGH ORDER HARMONIC GENERATION AND ATOMIC STABILIZATION IN ULTRA-INTENSE LASER PULSES
}

\author{
A. Maquet, T. Millack and V. Véniard \\ Laboratoire de Chimie Physique - Matière et Rayonnement \\ Université Pierre et Marie Curie \\ 11, rue Pierre et Marie Curie, 75231 Paris CEDEX 05, France
}

\begin{abstract}
We present results of recent numerical simulations on the non-linear response of a single-electron atom submitted to an intense laser pulse. We address both the questions of the light scattered by the atom and the possible stabilization against ionization. One of the results of our simulations is the the presence of hyper-Raman lines in the high-order harmonic spectra. We suggest that these lines could be a signature of atomic stabilization.
\end{abstract}

PACS numbers: $32.80 . \mathrm{Rm}$, 42.65.Ky

IIigh-order harmonic generation by rare gas atomic targets and the possible stabilization of atoms in the presence of ultra-intense laser pulses have emerged, since the late eighties, as new topics in the field of multiphoton physics. Before, it was commonly accepted in the community that a bound atomic system could never experience the strong field strengths needed to observe these processes. More precisely, experimental evidences seemed to indicate that the higher the laser field strength, the larger the multiphoton ionization probabilities. It was observed in fact that, for laser pulses with durations comprised between $10^{-9} \mathrm{~s}$ and a few picosecond, saturation occurred for ionization, i.e. a whole atomic sample was ionized in the higher intensity range. Accordingly, it was suggested that in an ultra-intense pulse the atoms would be ionized well before the intensity had reached its maximum [1]. It is interesting to note that, were this picture be correct, it would have implied that most multiphoton processes could be described within a mostly perturbative framework, as the non-perturbative regime could never be experienced by actual bound atomic systems. A consequence of this scenario would have been that only a few harmonics of the laser frequency, with exponentially decreasing intensities, could be generated by a strongly laser-driven atomic system.

It is in the late eighties that it was first shown experimentally $[2,3]$ that, in fact, the harmonic spectra do not display such a behaviour and that, on the contrary, they are characterized by a conspicuous plateau which can extend well beyond the 100th harmonic and is often followed by an abrupt cut-off [4]. Understandably, this has attracted a lot of attention and many theoretical simulations, 
quantum as well as classical, have been performed attempting to account for the main features of the harmonic spectra, recent reviews can be found in $[5,6]$. As a result of this intense theoretical activity, the origin of the plateau and the position of the cut-off are now mostly understood through the help of an interesting semi-classical model [7-9]. It has been shown that, in particular, the maximum photon energy in the harmonic spectrum is approximately given by the simple formula

$$
N_{\max } \omega_{L} \approx I+3 U_{p}
$$

where $I$ is the ionization energy of the atomic target and $U_{p}=F_{0}^{2} / 4 \omega_{L}^{2}$, with $F_{0}$ and $\omega_{L}$ being the laser field strength and frequency respectively, is the quiver energy of a free electron in the field. The quantity $3 U_{p}$ represents in fact the maximum kinetic energy an electron, initially at rest, can acquire in a laser field [10]. Similarly, the quantity $I+3 U_{p}$ approximately represents the maximum energy which can be released by an electron, initially bound close to the nucleus, then strongly driven by the external field and returning back to the atomic ground state. Let us mention that more detailed numerical simulations indicate that a more precise formula is [7-9]

$$
N_{\max } \omega_{L} \approx I+3.2 U_{p} \text {. }
$$

It should be noted that this formula is valid when considering the response of a single atom and that, in actual experiments, propagation effects seem to notably alter this result, $N_{\max }$ being significantly reduced [9]. Let us now turn to the second topic discussed here, namely the possible stabilization of atoms against ionization.

It is also after the mid-eighties that it was realized [11] that, in a high frequency regime, dressed atomic states lifetimes, after decreasing as expected with increasing field intensities, could reach a minimuria and then increase again as the field strength keeps growing. This was first demonstrated and made clear through the use of the so-called Kramers-IIenneberger transformation which certainly helped to introduce the picture of the dichotomy of the atomic system in an ultra-intense, high frequency, laser field: in the accelerated reference frame attached to the motion of the electron, this latter experiences a time-averaged modified Coulomb potential exhibiting a characteristic double-well structure with smoothed Coulomb singularities. The two minima of the average potential are separated by a distance of $2 \alpha_{0}$, where $\alpha_{0}=F_{0} / \omega_{L}^{2}$ is the amplitude of the quiver motion of a free electron in the laser field. Numerical simulations, showing stabilization in the ultra-high intensity regime, performed first for this approximate model, have been subsequently confirmed by a full Floquet-Schrödinger analysis [12]. It should be noted that the preceding analysis is only valid in adiabatic conditions in which atomic lifetimes can be defined. It has been also realized that it might be extremely difficult to meet the criteria for adiabaticity in the regime of ultra high intensities as the pulse turn-on should be kept slow, thus obliging the system to withstand intense fields for very long times [13]. This has motivated another set of theoretical simulations to analyse the response of model atoms to laser pulses with finite durations. The main outcome of these "numerical experiments" has been to show that atomic stabilization might be observed in more realistic situations, namely at frequencies comparable to the characteristic ionization frequency of the atom. 
In contrast with high-order harmonic generation, which has been observed in experiments well before, numcrical simulations were able to qualitatively reproduce the observations, atomic stabilization has been first predicted through theoretical analysis $[11,12,14,15]$. The direct experimental verification i.e. a decrease in the ionization yield for increasing laser intensities, which has been announced only very recently [16], is extremcly difficult and can be assessed only under very special circumstances (circular Rydberg states, for instance). We wish to suggest here that the signature of atomic stabilization might be found in harmonic spectra. More precisely, the presence of the hyper-Raman lines, associated to real incoherent light scattering transitions not ending into the atomic ground state and distinct from the harmonic lines, would reveal the existence of long-lived dressed atomic states [17]. We discuss next the occurrence of these lines in harmonic spectra.

Hyper-Raman lines are present in many numerical simulations of single atom spectra. We show here the results of numerical simulations performed in various strongly driven systems. The spectra have been obtained from a standard Fourier analysis of the time-dependent atomic dipole, either under the clectric dipole form or under the acceleration form. In the figures we show the power spectra associated to the following strongly driven anharmonic systems:

(a) 2-level system [18], Fig. 1;

(b) classical driven anharmonic oscillator [19], Fig. 2;

(c) classical Kepler system, Fig. 3;

(d) 1-dimension soft-Coulomb system [17], Fig. 4.

In quantum simulations, the origin of these hyper-Raman or incoherent lines can easily be traced back to real transitions between the Floquet states of the dressed atom. This is particularly clear in the simpler case of a 2-level atom as shown in Fig. 5. The more involved situation of a model potential supporting an infinite number of bound states plus a continuum has been discussed in [17] and will be briefly presented below. Another recent discussion may be found in [20].

In classical simulations, the corresponding lines can be associated to sumand difference-combinations of the laser frequency and of the (ac-shifted) characteristic frequency of the system (anharmonic oscillator or Kepler). Note, however, that it is in general very difficult to compute the positions of these lines at the field strengths (comparable with the scaled atomic field strength) required to observe high order harmonics. Only perturbative estimates, valid in the low intensity regime, are available and can be easily assessed [21].

An interesting common feature of these simulations is that hyper-Raman lines can disappear from the power spectra if a phenomenological damping term is included into the equations of motion of the system. This is easily performed in the case of the quantum 2-level system and for the classical anharmonic oscillator (see Figs. $1 \mathrm{~b}$ and $2 \mathrm{~b}$ ). In more realistic models featuring a continuous, positive energy spectrum (classical Kepler and 1-dimensional soft-Coulomb systems) ionization is the process which dominantly contributes to the width of the states and can be associated to a damping mechanism. On the other hand, in a classical perspective, it clearly appears that, starting from a microcanonical ensemble of trajectories, the ones leading to ionization in the presence of the field no longer contribute to the scattered radiation spectrum (except for a trivial Thomson component), as 

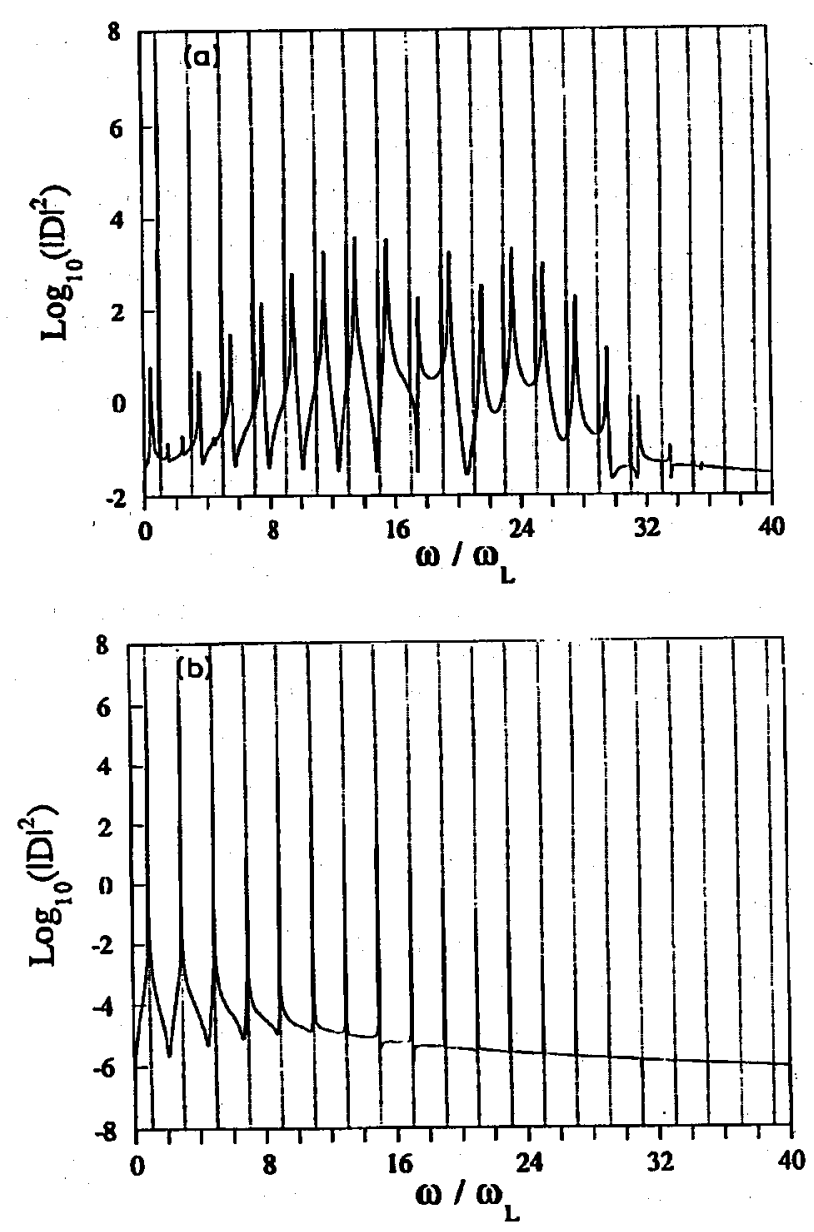

Fig. 1. Power spectrum of the dipole for a two-level atom. The atomic frequency is $\omega_{0}=1$ a.u., the laser frequency is $\omega_{L}=0.086$ a.u. and the Rabi frequency $\Omega=1$ a.u. Part (a) corresponds to a system without damping while part (b) corresponds to a system with damping.

soon as the electron leaves the vicinity of the nucleus [22].

One consequence of this analysis is that, as already noted in several instances, multiphoton ionization (ATI) and high-order harmonic generation, though taking place at the same intensity range, are competing processes. This means more precisely that from a given finite atomic sample, the harmonic intensities should decrease in time as the ionization yield grows. It should be noted however that the surviving ionic core with more tightly bound electrons remains available to generate harmonics, albeit the higher laser field strength [23]. Another aspect of this competition is that, as already pointed out by Knight et al. [24], if atoms are stabilized against ionization, they can remain available for high order harmonic generation in a very high intensity regime thus presumably leading to enhanced harmonic spectra. 

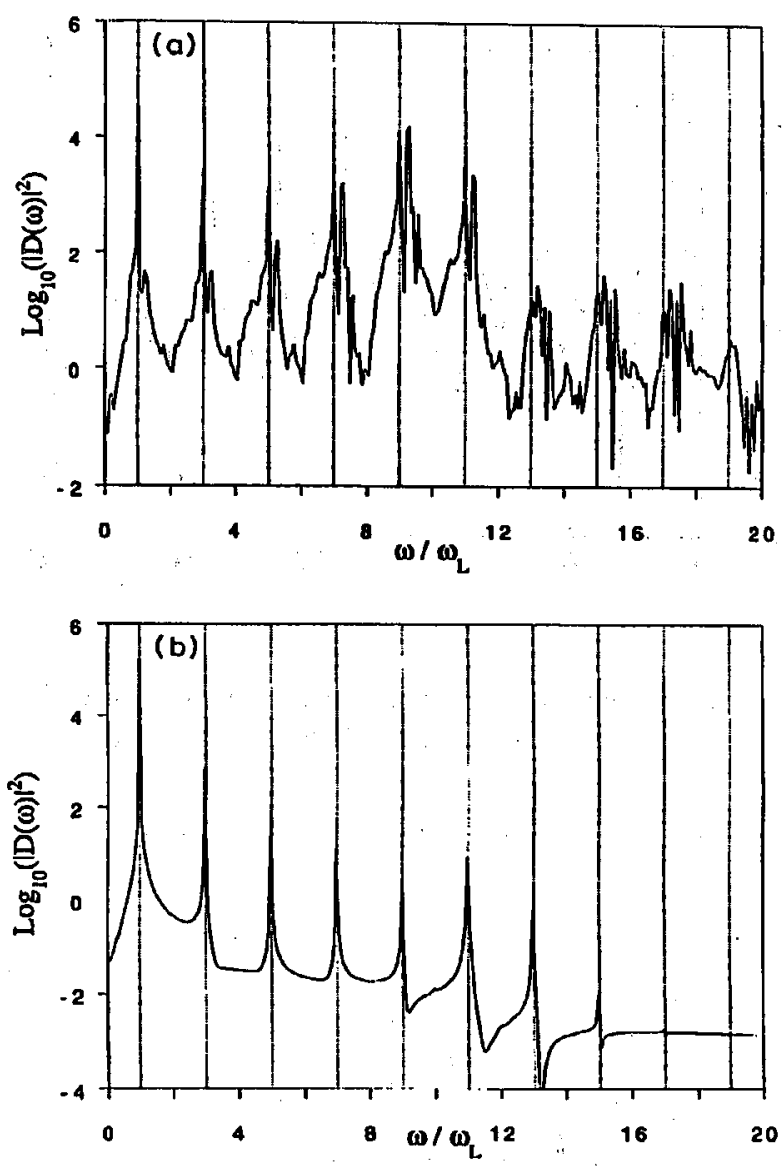

Fig. 2. Power spectrum of the averaged dipoles for a classical driven anharmonic (quartic) oscillator. The anharmonicity is $\lambda=-1 / 16$, the laser frequency is $\omega_{L}=0.086$. Part (a) corresponds to a system without damping, the electric field being $F_{0}=0.296$ a.u. while part (b) corresponds to a system with damping with $F_{0}=0.7$ a.u.

Another interesting featur of this balance between ionization and harmonic generation is that the presence of sharp hyper-Raman lines embedded in harmonic spectra could indirectly indicate the existence of stabilized atomic states. This observation emerged from numerical simulations performed on the harmonic spectrum from a strongly 1D soft-Coulomb model atom. We have observed that, in some cases, the spectrum radiated by the single-atom forced dipole could contain very distinctive hyper-Raman lines, see for instance Fig. 4. This spectrum has been obtained for a relatively low-frequency field $(\omega=0.08$ a.u., to be compared to the characteristic ionization frequency of the bare atom $\omega_{0}=0.667 \mathrm{a} . \mathrm{u}$.) with a quite high constant amplitude $\left(F_{0}=0.058\right.$ a.u.) [17]. We have been able to assign these lines to real transitions between dressed Floquet atomic levels, adiabatically connected to the ground state and to the $(n=3)$ excited state, respectively, see 


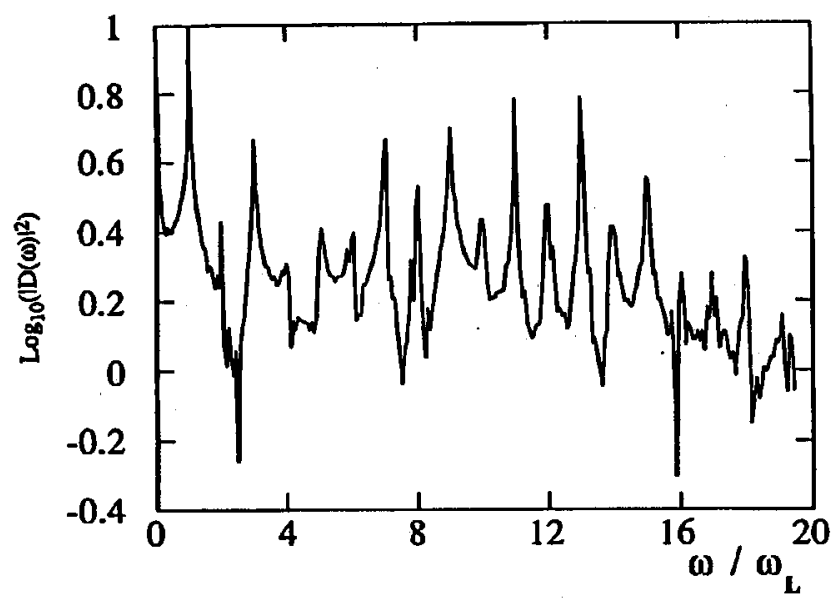

Fig. 3. Power spectrum of the dipole for a classical ground state hydrogen atom. The laser frequency is $\omega_{L}=0.086$ a.u. and the electric field strength is $F_{0}=0.12$ a.u.

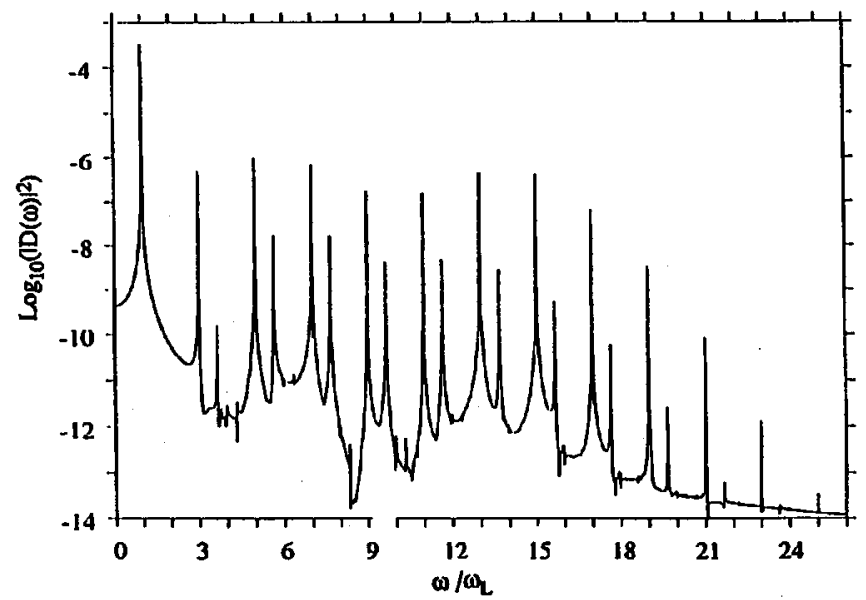

Fig. 4. The scattered light spectrum for a $1 \mathrm{D}$ soft-Coulomb model at a constant field strength $F_{0}=0.058$ a.u. after a turn on of 320 laser cycles with a frequency $\omega=0.08$ a.u. The system was initially in the ground state.

the Floquet spectrum, Fig. 6. Another interesting feature of the Floquet spectrum is that the width (i.e. the imaginary part) of the corresponding "excited" state depends in a complicated way on the laser field strength, as shown in Fig. 7. After increasing, as expected, with the field strength, its width starts again to decrease around $F_{0}=0.03$ and becomes extremely small at $F_{0}=0.058$. This small width is responsible for the sharpness of the hyper-Raman features observed in the spectrum of Fig. 4. It is also a clear signature of the stabilization of the corresponding 


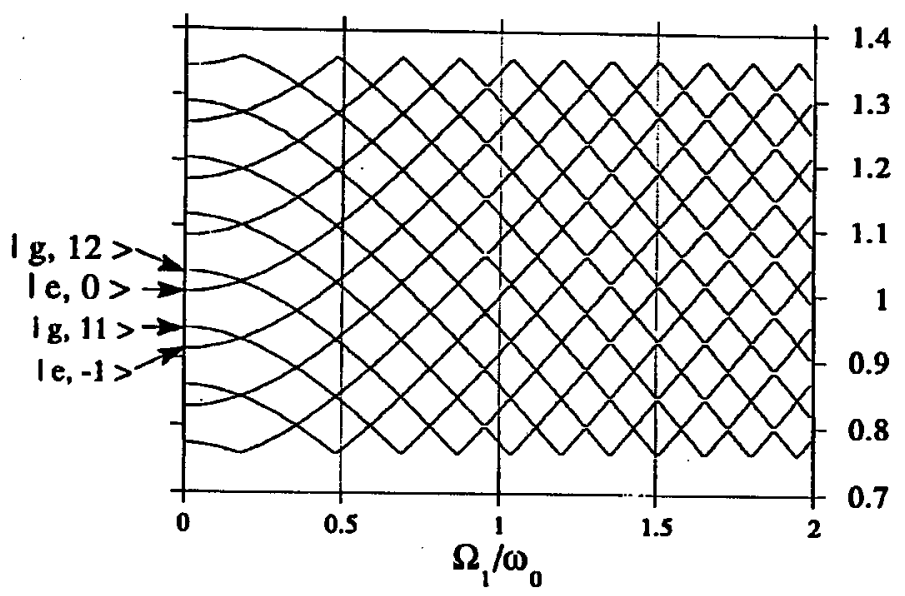

Fig. 5. Floquet states energies for a two-level atom as a function of the Rabi frequency, the laser frequency being $\omega_{L}=0.086$ a.u. and the atomic frequency being $\omega_{0}=1$ a.u.

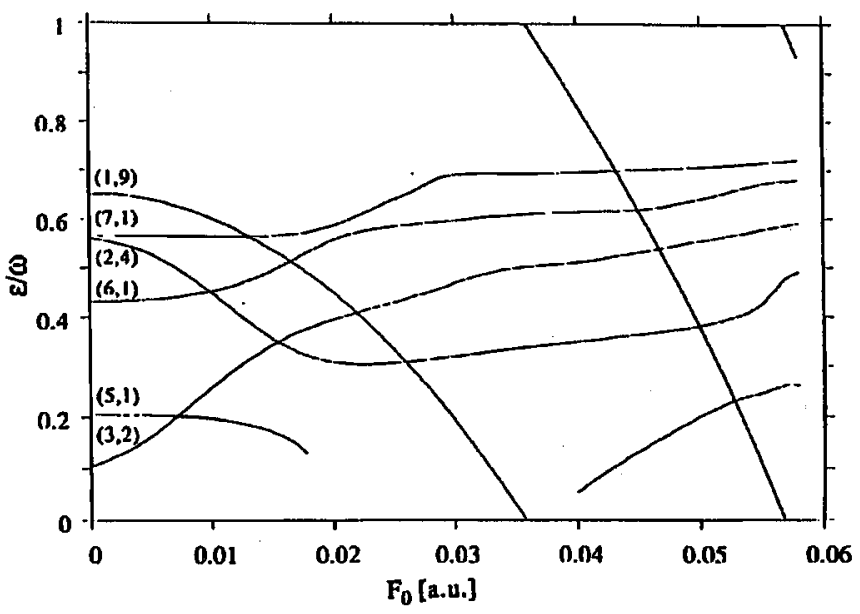

Fig. 6. The positions of the Floquet resonance states as a function of the field strength for the soft Coulomb potential. The different states are labeled $(n, k)$ where $n$ denotes the principal quantum number of the unperturbed state and $k$ is the number of photons to be added to the unperturbed energy to reach $\left[0, \omega_{L}\right]$.

state in the presence of the field.

It should be stressed that the preceding analysis is only valid for a constant amplitude field. In the more realistic situation corresponding to a field with a time-varying envelope, as the dressed atomic levels continuously cliange in a complicated fashion with the field strength, it is likely to observe broader features, as shown in Fig. 8.

Another important aspect regarding the possible observation of these lyyper-Raman lines is that they are essentially incoherent, i.e. they will not add 


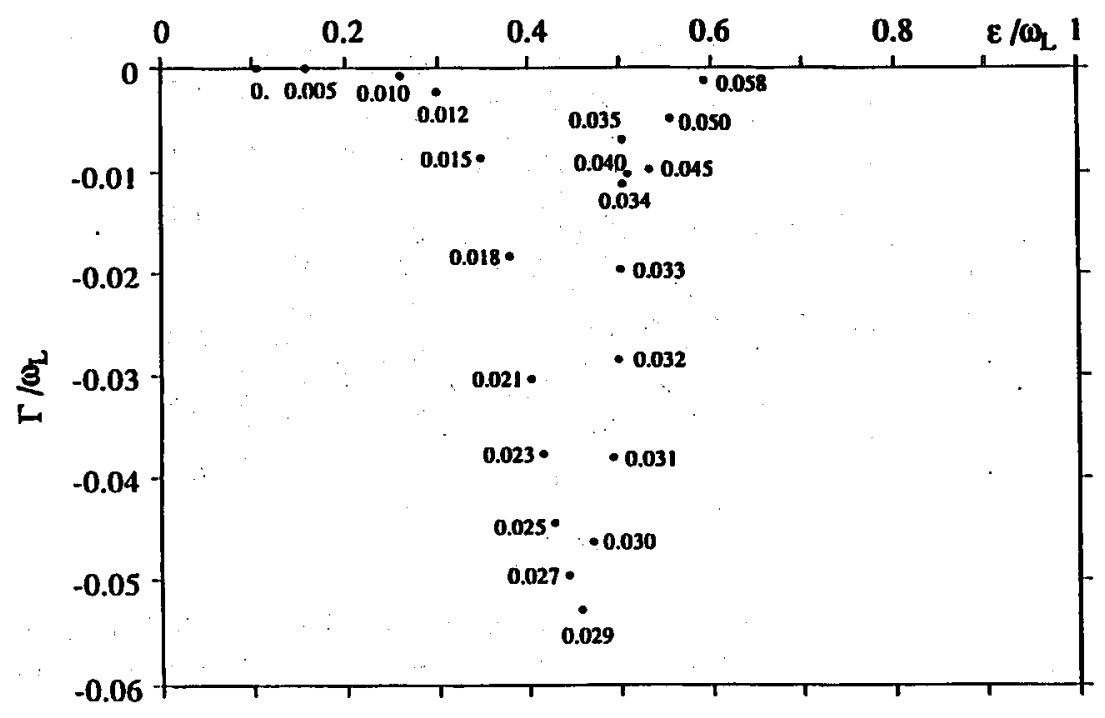

Fig. 7. Location of the $n=3$ resonance state in the complex energy plane as a function of the field strength for a frequency of $\omega_{L}=0.08$ a.u. The numbers indicate the field strength in atomic units.

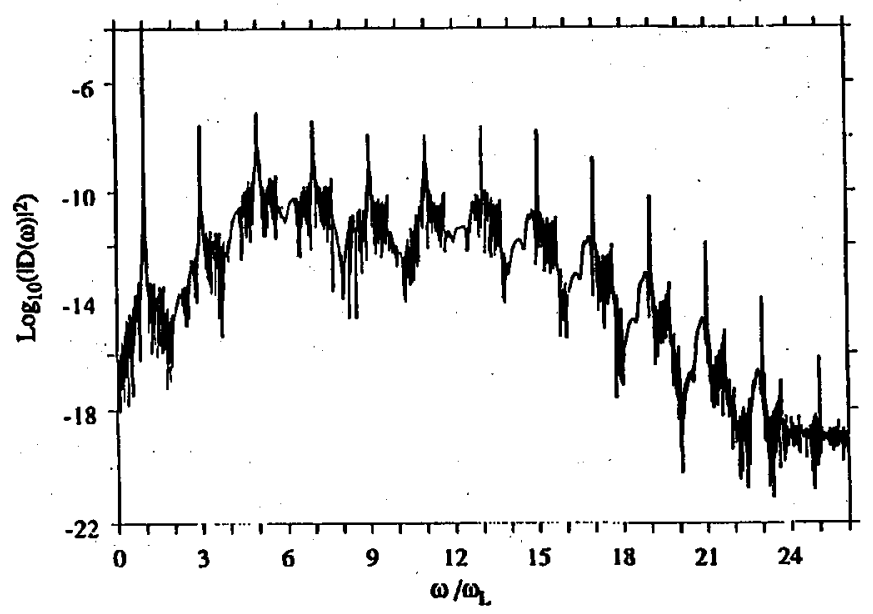

Fig. 8. The scattered light spectrum produced by a 640 laser cycle pulse with frequency of $\omega_{L}=0.08$ a.u. and a maximum field strength of $F_{0}=0.058$ a.u.

coherently in the forward direction as harmonics do in macroscopic samples. Accordingly, their relative intensities will grow only as $N$, the number of active atomic centers in the sample, instead of $N^{2}$ for harmonics. However, as already noted by LaGattuta [20], they might be detected at another observation angles than the forward direction. 


\section{References}

[1] P. Lambropoulos, Phys. Rev. Lett. 20, 2141 (1985).

[2] A. Mac Pherson, G. Gibson, H. Jara, U. Johann, T.S. Luk, I. McIntyre, K. Boyer, C.K. Rhodes, J. Opt. Soc. Am. B 4, 595 (1987).

[3] M. Ferray, A. L'IIuiller, X. Li, L.A. Lompré, G. Mainfray, C. Manus, J. Phys. B 21, L31 (1988).

[4] A. L'Huiller, Ph. Balcou, Phys. Rev. Lett. 70, 774 (1993).

[5] A. L'Huiller, K.J. Schafer, K.C. Kulander, J. Phys. B 24, 3315 (1991).

[6] A. L'Huiller, A.L. Lompré, G. Mainfray, C. Manus, in: Advances in Alomic, Molecular and Optical Physics, Suppl. 1, Ed. M. Gavrila, Academic, New York 1992, p. 139.

[7] K.C. Kulander, in: Proc. Workshop SILAP III, Eds. B. Piraux, A. L'Huiller, K. Rzążewski, Plenum Press, New York, in press.

[8] P.B. Corkum, to be published.

[9] M. Lewenstein, Ph. Balcou, M.Yu. Ivanov, A. L'Huiller, P.B. Corkum, to be published.

[10] T.F. Gallagher, Phys. Rev. Lett. 61, 2304 (1988).

[11] M. Gavrila, J.Z. Kaminski, Phys. Rev. Lett. 52, 614 (1984); M. Pont, N.R. Walet, M. Gavrila, C.W. Mc Curdy, Phys. Rev. Lelt. 61, 939 (1988); M. Pont, M. Gavrila, Phys. Rev. Lett. 65, 2362 (1990); M. Pont, N.R. Walet, M. Gavrila, Phys. Rev. A 41, 477 (1990).

[12] R.M. Potvliege, P.H.G. Smith, Phys. Rev. A 48, R46 (1993).

[13] V. Véniard, A. Maquet, T. Menis, in: Proc. Workshop SILAP III, Eds. B. Piraux, A. L'Huiller, K. Rzążewski, Plenum Press, New York, in press.

[14] K.C. Kulander, K.J. Schafer, J.L. Krause, in: Advances in Atomic, Molecular and Optical Physics, Suppl. 1, Ed. M. Gavrila, Academic, New York 1992; J.H. Eberly, R. Grobe, C.K. Law, Q. Su, idem.

[15] J. Grochmalicki, M. Lewenstein, K. Rzążewski, Phys. Rev. Lett. 66, 1038 (1991); R. Grobe, C.K. Law, Phys. Rev. A 44, 4114 (1991); T. Menis, R. Taïb, V. Véniard, A. Maquet, J. Phys. B 25, L263 (1992); M. Gajda, J. Grochmalicki, M. Lewenstein, K. Rzążewski, Phys. Rev. A 46, 1638 (1992); L. You, J. Mostowski, J. Cooper, Phys. Rev. A 45, 3203 (1992).

[16] H.G. Muller et al., to-be published.

[17] T. Millack, A. Maquet, J. Mod. Opt., 1993, in press.

[18] R. Taib, Ph.D. Thesis, Université Pierre et Marie Curie, 1992, unpublished.

[19] T. Menis, Ph.D. Thesis, Université Pierre et Marie Curie, 1993, unpublished.

[20] K.J. LaGattuta, Phys. Rev. A 48, 666 (1993).

[21] L. Locascio, A. Maquet, T. Menis, V. Véniard, unpublished.

[22] G. Bandarage, A. Maquet, T. Menis, R. Taïb, V. Véniard, J. Cooper, Phys. Rev. $A$ 46, 380 (1992).

[23] See the proceedings of the workshop SILAP III, Eds. B. Piraux, A. L'Huiller, K. Rzążewski, Plenum Press, New York, in press.

[24] V.C. Reed, K. Burnctt, P.L. Knight, to be published in Phys. Rev. A. 